\title{
Beyond cognitive-behavioral therapy for fibromyalgia: addressing stress by emotional exposure, processing, and resolution
}

\author{
Mark A Lumley* \\ See related research by Alda et al., http://arthritis-research.com/content/13/5/R173
}

\begin{abstract}
I comment on the well-designed trial by Alda and colleagues reported in a recent issue of Arthritis Research and Therapy which demonstrated some benefits of cognitive-behavioral therapy (CBT) for fibromyalgia (FM). CBT in this and other studies provides statistically significant but rather modest benefits for FM. This may be because CBT does not directly address the high rates of victimization, posttraumatic stress disorder, and emotional avoidance experienced by a substantial number of patients with FM. Interventions that encourage emotional exposure, processing, and resolution of stressful or traumatic experiences and relationships hold potential for larger effects for many patients and need to be tested.
\end{abstract}

Alda and colleagues [1] present a well-designed and implemented randomized trial (RCT) comparing cognitive-behavioral therapy (CBT), pharmacotherapy, and treatment-as-usual for patients with fibromyalgia (FM). I commend their inclusion of a pharmacotherapy comparison, assessment of mediators and outcomes, and 6-month follow-up. CBT improved several outcomes, adding to a growing literature supporting CBT for FM.

But how well does CBT work for FM? A critical look at this and similar studies shows that the benefits - although non-zero - are rather modest clinically. The largest effects in this study (approximately one standard deviation compared with treatment-as-usual) were for pain catastrophizing and acceptance. But these are actually change processes or mediators directly targeted by CBT, not outcomes of clinical interest. In contrast, there were

*Correspondence: mlumley@wayne.edu

Department of Psychology, Wayne State University, Detroit, MI 48202, USA

BioMed Central o 2011 BioMed Central Ltd moderate-sized effects on overall FM impact and quality of life, small effects on depression and anxiety, and - of greatest concern - no effects on pain. These results are generally consistent with recent meta-analyses [2,3], which report non-zero but rather modest benefits of CBT for FM. Overall, it appears that only a minority of FM patients - perhaps one-third - demonstrate clinically meaningful improvement from CBT and other psychological/behavioral interventions [4].

Are small to moderate effects and a minority of patients improving the limit of effectiveness of psychological interventions for FM? Do genetics, long-term central nervous system sensitization, and socioeconomic contingencies simply 'account for more variance' and trump the influence of psychological processes? Perhaps, but I argue that we do not yet know, because our intervention efforts have not been guided by the larger literatures on effective psychological therapies and pathological processes in FM.

I find a tendency, especially in medical settings, to equate CBT with 'effective psychological therapy' and to contrast it with one alternative - 'talk therapy'. This is incorrect. CBT is one of many psychological interventions that have proliferated over the past few decades, many of which are active, time-limited, and - most importantly - beneficial. Furthermore, psychotherapy research has identified general processes that predict positive outcomes across a range of psychological therapies. Five such processes are: a) providing a new rationale for the problem and how to change it; b) teaching symptom and self-management skills; c) experiencing and processing avoided emotions and memories; d) encouraging behaviors that have been avoided, usually due to negative emotions (for example, fear, guilt); and e) providing a supportive therapeutic relationship that also corrects faulty interpersonal expectations [5]. The primary focus of CBT for FM is providing the rationale of, and teaching cognitive and behavioral skills for, symptom management. CBT for pain typically does not 
'open up' negative emotions, encourage previously avoided emotional experiences (except pain exposure exercises, which are probably helpful), or use the therapeutic relationship as a change vehicle.

How is this relevant to FM? Many studies have found elevated rates of trauma, victimization, and interpersonal conflict among people with FM - at least among patients actively seeking treatment [6]. Such trauma and the subsequent avoidance of emotional processing lead to the increased post-traumatic stress disorder found in FM [7]. Suppressed and dysregulated anger, emotional unawareness and confusion, and reactivity to interpersonal conflict are increased in FM. The elevated pain catastrophizing targeted by Alda and colleagues [1] encompasses rumination, helplessness, and somatic magnification common consequences of unresolved stress.

Should we target for treatment the unresolved stress in patients with FM? If so, how? Interestingly, effective treatments for trauma and post-traumatic stress disorder, which encourage experiencing, expressing, and processing of stress-related emotional memories, remain largely untested for those FM patients who have victimization histories and emotional avoidance [8]. There are a handful of small trials indicating the benefits to people with FM of private writing about stress, group therapy for enhancing emotional awareness, and individual therapy targeting unresolved stressors [9]. It is noteworthy that Alda and colleagues actually included two ancillary exercises that activate avoided emotions expressive writing and assertive communication. I applaud this, but encourage testing of interventions that have emotional processing as a primary target.

Perhaps we fear that patients will respond negatively to such an intervention - rejecting it, feeling stigmatized, and having increased symptoms. Such interventions also are emotionally challenging for therapists. We should not, however, let our fears prompt avoidance of potentially adaptive experiences. Colleagues and I are testing an intervention that has FM patients confront and process avoided emotional experiences and relationships, and are comparing it to CBT and an educational control. We do not yet know this intervention's effects, how it compares with CBT, and importantly - given the heterogeneity of FM - which patients benefit most from each approach [10]. However, our initial observations are that almost all of our patients acknowledge that stress contributes to their FM symptoms, and patients find that confronting avoided emotions immediately influences their pain, which powerfully demonstrates the relevance of their emotions. I encourage researchers, clinicians, and patients to be courageous and develop, test, and - if empirically supported - implement interventions that directly address the unresolved stressors experienced by many patients with FM.

Abbreviations

CBT, cognitive-behavioral therapy; FM, fibromyalgia.

Competing interests

The author declares that he has no competing interests.

Published: 1 December 2011

References

1. Alda M, Luciano-Devis JV, Andrés E, Serrano-Blanco A, Rodero B, López del Hoyo Y, Roca M, Moreno S, Magallón R, García-Campayo J: Effectiveness of cognitive behaviour therapy for the treatment of catastrophisation in patients with fibromyalgia: a randomised controlled trial. Arthritis Res Ther 2011, 13:R173

2. Bernardy K, Füber N, Köllner V, Häuser W: Efficacy of cognitive-behavioral therapies in fibromyalgia syndrome - a systematic review and metaanalysis of randomized controlled trials. J Rheumato/ 2010, 37:1991-2005

3. Glombiewski JA, Sawyer AT, Gutermann J, Koenig K, Rief W, Hofmann SG: Psychological treatments for fibromyalgia: a meta-analysis. Pain 2010, 151:280-295.

4. Turk DC: The potential of treatment matching for subgroups of patients with chronic pain: lumping versus splitting. Clin J Pain 2005, 21:44-55.

5. Lambert MJ, Ogles BM: The efficacy and effectiveness of psychotherapy. In Handbook of Psychotherapy and Behavior Change. 5th edition. Edited by Lambert MJ. New York: Wiley; 2004:139-193.

6. Häuser W, Kosseva M, Üceyler N, Klose P, Sommer C: Emotional, physical, and sexual abuse in fibromyalgia syndrome: A systematic review with metaanalysis. Arthritis Care Res 2011,63:808-820.

7. Yunus MB: Fibromyalgia and overlapping disorders: the unifying concept of central sensitivity syndromes. Semin Arthritis Rheum 2007, 36:339-356.

8. Leserman J: Sexual abuse history: Prevalence, health effects, mediators, and psychological treatment. Psychosom Med 2005, 67:906-915.

9. Lumley MA, Cohen JL, Borszcz GS, Cano A, Radcliffe A, Porter L, Schubiner H, Keefe FJ: Pain and emotion: A biopsychosocial review of recent research. J Clin Psychol 2011, 67:1-27.

10. Van Houdenhove B, Luyten P: Customizing treatment of chronic fatigue syndrome and fibromyalgia: the role of perpetuating factors. Psychosomatics 2008, 49:470-477.

doi:10.1186/ar3511

Cite this article as: Lumley MA: Beyond cognitive-behavioral therapy for fibromyalgia: addressing stress by emotional exposure, processing, and resolution. Arthritis Research \& Therapy 2011, 13:136. 